\title{
Diabetes Mellitus en 93 enfermos esquizofrénicos crónicos
}

\author{
Dan Cohen $\mathrm{PhD}$ * \\ Barteld Puite ${ }^{\star *}$ \\ Jack Dekker $\mathrm{PhD}^{\star \star *}$ \\ Christine Gispen de Wied ${ }^{\star \star \star}$
}

* Director del Departamento de Largas

Estancias Rijngeestgrouup en

Noordwijk/Noordwijkerhout

** Estadístico en el Departamento de Investigación y Desarrollo de Mentrum

*** Psicólogo/investigador en la Organización de Salud Mental y Cuidados de Mentrum, Amsterdam

**** Director de Investigación del Centro Médico Universitario de Utrecht, Departamento de Psiquiatría

HOLANDA

RESUMEN - Objetivos: A partir de una Investigación exploratoria sobre la hiperglicemia o Diabetes Mellitus, tipo 2, entre esquizofrénicos crónicos en una sala de un hospital general psiquiátrico, a todos los enfermos con diagnóstico de esquizofrenia o trastornos psico-afectivos se les preguntó si querían colaborar en el estudio.

Métodos: Hiperglicemia o DM, tipo 2, en pacientes no ayunadores que los fueron diagnosticados según las directrices del NHG (1999). 93 de los 99 enfermos esquizofrénicos o psico-afectivos participaron en la investigación.

Resultados: La hiperglicemia se diagnosticó en 11 de los 93 pacientes, de los cuales en unicamente uno se conocía su existencia. La prevalencia de la hiperglicemia en edades comprendidas entre los 20 y los 49 años resultó importante en la muestra tomada $(15,2 \%$ vs $5,7 \%, \mathrm{OR}=2,959, \mathrm{CI}=1,230-7,119, \mathrm{p}<0,05)$. La presencia general de DM, tipo 2, fue alta en comparación con la muestra general $(7,5 \%$ vs $1,9 \%, \mathrm{OR}=4,288, \mathrm{CI}=1,979-9,289$, $\mathrm{p}<0,001)$. A pesar de existir un incremento importante de obesidad en la muestra (20\% vs $8 \%, \mathrm{OR}=2,953, \mathrm{CI}=1,698, \mathrm{p}<0,001$ ), no existía relación alguna con la importante prevalencia de hiperglicemia o DM, tipo 2, ni tampoco relación entre el trastorno diabético y la medicación antipsicótica recetada.

Conclusiones: En el estudio exploratorio de 93 enfermos esquizofrénicos, se encontró una prevalencia importante de hiperglicemia y Diabetes Mellitus tipo 2, lo cual no podía ser explicado por la significativa presencia de sobrepeso. Contrastándolo con recientes estudios, no se encontró relación alguna entre trastorno diabético y medicación antipsicótica recetada. Que esto se deba a diferencias en las poblaciones o a otras diferencias meto- 
dológicas, merece un estudio mas detallado. Este estudio tiene tres grandes limitaciones. Una es el impacto de la inactividad, factor de riesgo bien conocido en la Diabetes Mellitus tipo 2, y que no se valoró. La segunda limitación es la selección de la población de estudio: únicamente se incluyeron esquizofrénicos crónicos en el estudio. La tercera limitación es la dificultad de valorar el efecto mantenido de un régimen antipsicótico durante largos decenios a base de diferentes medicamentos antipsicóticos y con diferentes dosis sobre la homeostasis de la glucosa. Por todo ello son necesarias muchas mas investigaciones para esclarecer estas cuestiones.

\section{Introducción}

La cuestión de si los pacientes esquizofrénicos sufren o no un mayor riesgo de desarrollo de DM, tipo 2 que el resto de la población en general, era ya bien conocida para la psiquiatría. En 1899, mucho antes de la introducción del tratamiento antipsicótico, Henry Maudsley escribía en La patología de la mente que "La Diabetes es una enfermedad que a menudo aparece en familias en las que predomina la poca higiene" (Mukherjee 1989). Entre los neurólogos holandeses pioneros en este área encontramos a Heidema, quien publicó una tesis titulada: Niveles de glucosa como determinantes de enfermedades neurológicas y psiquiátricas en 1918, así como Kooy quien escribió un elaborado ensayo titulado La hiperglicemia en los trastornos mentales en 1919. La gran cantidad de trabajos escritos con anterioridad a la Segunda Guerra Mundial acerca de este tema concluían sobre el hecho de que los enfermos psiquiátricos, bajo el efecto de la glucosa, sufrieran mas a menudo de hiperglicemia, y por largos periodos de tiempo que otros enfermos (Mc Farland 1938). Unos pocos años después de la introducción de la clorpromacina, en 1952 (Delay 1952), empiezan a aparecer casos de irrupción de diabetes provocados por el uso de clorpromacina (Charatan 1955, Hiles
1956). Estos informes se centran en la detección de poblaciones hospitalarias tratadas con antipsicóticos en las que se producían estas situaciones (Amdisen 1964, Clayer 1967, Keskiner 1973, Waitzkin 1966a 1966b). Los resultados -con un claro predominio de investigaciones americanas- se describen en el estudio de Thonnard-Neumann. En este estudio, en una muestra de 405 mujeres enfermas y durante un seguimiento de diez años, se observó el incremento de la presencia de DM, tipo 2, de 4,2\% a 17,2\%. En 1966 más investigaciones, entre ellas un estudio de Schwarz y Munoz (1968), y con una muestra de 850 enfermos psiquiátricos, proporcionaron la única evidencia que contradecía a los resultados anteriormente mencionados: La prevalencia de DM (2,5\%) e hiperglicemia $(3,2 \%)$ como establecieron Schwarz y Muñoz, se hallaba dentro de los valores límites de la población general.

Los tests realizados en animales demuestran como de las cuatro fenotiazinas examinadas, la clorpromazina es la causante de los incrementos mas altos de los niveles de glucosa la sangre (Proakis 1971). Sin embargo, el mecanismo es mas complejo involucrando factores tales como la afectación de la secreción de la insulina (Bonacorssi 1964, Ammon 1973) la reducción de la utilización de la glucosa periférica (Bhide 1965, Ghafghazi 1968, Jori 1966) la estimu- 
lación de los receptores B-andrenérgicos (la clorpromazina o la hiperglicemia inducida por la adrenalina puede ser antagonizada por sustancias betabloqueantes, Ghafghazi 1968, Jori 1966) y los efectos sobre el sistema de control central, de por ejemplo, las glándulas suprarrenales (Wannarka 1983).

Varios estudios recientes acerca de la presencia de la Diabetes Melitus en pacientes esquizofrénicos tratados con antipsicóticos clásicos, mostraron un incremento importante de la prevalencia. Un estudio italiano (Mukherjee 1966) en 95 pacientes esquizofrénicos, de entre 45 y 74 años de edad, mostró una prevalencia de diabetes mellitus tipo 2 del 15,5\%, considerablemente mas alto que el $2,1 \%$ - 3,2\% presente en la población general. En cuanto a la variable edad, mostró un 12,9\% de prevalencia de Diabetes Mellitus en enfermos con edades de entre 50 y 59 años, y un 18,9\% para enfermos con edades de entre 60 y 69 años. Estos porcentajes correspondientes a estos grupos de edades representan para la población general un $3 \%$ y un $5,5 \%$ respectivamente. Un estudio americano sobre 278 enfermos esquizofrénicos que habían sido dados de alta recientemente (Mukherjee 1995) mostró una proporción de DM, tipo 2 del $16,2 \%$, variando desde un $1,6 \%$ en los pacientes menores de 40 años, a un $25 \%$ en los mayores de 70 años. Los mismos investigadores encontraron una prevalencia de DM, tipo 2, del 24,5\% en un estudio realizado en un grupo de 98 pacientes procedentes de una policlínica, con un incremento que iba desde $0 \%$ en los pacientes menores de 40 años, hasta un $50 \%$ en los mayores de 70 años (Mukherjee 1995).

En cuanto a la cuestión de la irrupción de la diabetes asociada a la esquizofrenia se ha renovado el interés desde 1994, con la particular atención puesta en la clozapina (Kamran 1994, Colli 1999, Brugman et al. 2000) y en la olanzapina (Fertig 1998, Wirshing 1998, Lindenmayer 1999). En Suecia la prevalencia de la afectación de la tolerancia a la glucosa (IGT, determinada sobre la base del Test de Tolerancia Oral a la Glucosa, OGTT) y a la DM, tipo 2, se estudió en 63 enfermos tratados con el antipsicótico atípico, clozapina y en otros 67 enfermos tratados con antipsicóticos típicos depot (Hägg 1998). La prevalencia resultante fue del $10 \%$ de IGT con clozapina y del 6\% de DM en el caso de los antipsicóticos depot, no existiendo diferencias significativas en cuanto al predominio de la DM, tipo 2, entre pacientes tratados con clozapina (12\%) y aquellos otros a los que se les trató con antipsicóticos depot (6\%).

En resumen, independientemente de las deficiencias metodológicas y de otras diferencias que pudieran aparecer, dos conclusiones se desprenden de los diferentes estudios:

1. Un incremento de la presencia de Diabetes Mellitus, tipo 2 en los esquizofrénicos en comparación con la población en general.

2. Este incremento aparece tanto en enfermos tratados con antipsicóticos típicos como con atípicos.

La segunda conclusión, la disrupción del metabolismo de la glucosa en los pacientes esquizofrénicos tratados con anti-psicóticos clásicos y atípicos puede ser explicado de dos maneras diferentes:

a) La posibilidad de un incremento de la sensibilidad para desarrollar DM, tipo 2, en pacientes que sufren de esquizofrenia (Mukherjee 1995).

b) La posibilidad de efectos asociados colaterales asociados a la medicación antipsicótica. 
En cuanto a esta última posibilidad, que se trate de un antipsicótico típico o no, no parece ser tan relevante como otros efectos, por ejemplo los serotonérgicos de los antipsicóticos (Wirshing 1998).

Teniendo en cuenta la anterior información como punto de partida, dentro del marco de una mejora de la calidad de los cuidados somáticos en los enfermos esquizofrénicos crónicos en ausencia de datos comparables relativos a la situación en Holanda, se inició un programa de investigación exploratorio en pacientes esquizofrénicos crónicos.

Los objetivos del estudio fueron describir la prevalencia de hiperglicemias y DM tipo 2 , en pacientes esquizofrénicos internados comparado ello con la población en general, con el fin de establecer:

a) La prevalencia de la hiperglicemia y la DM tipo 2.

b) La influencia de factores de riesgo tales como la edad, el sexo y el peso en relación con el predominio de la hiperglicemia y la DM, tipo2.

c) La influencia de los antipsicóticos tradicionales y los atípicos, en relación con la prevalencia de la hiperglicemia y la DM, tipo 2.

\section{Método}

\section{La Muestra}

Todos los pacientes que recibían asistencia en la Sala de Hospitalización de la Organización para la Salud Mental de Mentrum, Amsterdam, fueron diagnosticados mediante criterios DSM-IV, como enfermos que sufrían esquizofrenia o Trastornos psicoa- fectivos cumpliendo los requisitos para participar en el estudio. En aquellos casos donde existía alguna duda en cuanto a la precisión de su diagnóstico, se llevó a cabo una investigación mas exhaustiva tomando como base el historial del paciente (cartas de admisión y altas) y un perfil clínico actualizado. No había razón alguna para excluir adicionales diagnósticos somáticos o psiquiátricos, de modo que cuando se indicaba así, se incluían en el estudio como variables. Debido a sus carencias se consideraba esencial asegurar la inclusión máxima de esta población patológica tan especial, la cual supone realmente, un grupo difícil de incluir en cualquier estudio. Para lograr la máxima inclusión se procuró hacer las mínimas demandas a los pacientes. Consideramos que la demanda de comida (incluyendo café con leche y azúcar) en al menos diez horas, suponía un esfuerzo para este particular grupo de pacientes, así que se decidió por ello que los niveles de glucosa se determinarían en pacientes que no ayunasen. Como elemento adicional a esta decisión se redujeron las violaciones potenciales del protocolo (tanto si se informaban o no). En tanto que los resultados podían distorsionarse, la distorsión se reflejaría con una mayor probabilidad en una sobrestimación de la prevalencia (para mas detalle ver el análisis estratégico).

De los 158 pacientes internados en la Institución, 99 cumplían los criterios para ser incluidos en el estudio, solicitándoles su participación en el estudio. 93 de ellos estaban dispuestos a cooperar y permitieron que se les tomaran muestras de sangre. El último test fue realizado por el 59,2\% de los hombres $(\mathrm{N}=55)$ y por el $40,8 \%$ de las mujeres $(\mathrm{N}=38)$, correspondiendo a una media de edad de 51,3 años. Un enfermo varón padecía de hiperglicemia, y cinco mujeres y dos varones fueron diagnosticados de DM, tipo 
Tabla I

Medicamentos antipsicóticos prescritos

$\mathrm{N}^{\circ}{ }^{\circ}$ de paciente $(\mathrm{N}=93)$

Antipsicóticos tradicionales 39

Alimazina (Nedeltran)

Bromperidol (Impromen)

Flufenazina (Anatensol)

Flupentixol (Fluanxol)

Haloperidol (Haldol)

Penfluridol (Semap)

Perfenazina (Trilafon)

Pipamperon (Dipiperon)

Sulpiride (Dogmatil)

Thioridazina (Melleril)

Zuclopentixol (Cisordinol)

Antipsicóticos atípicos

atipicos -51

Clozapina (Leponex) $\quad 28$

Olanzapina (Zyprexa) $\quad 8$

Risperidona (Risperdal) $\quad 15$

Antipsicóticos divesos 1

No antipsicóticos $\quad 2$

2. La monoterapia antipsicótica se empleó en 92 de los 93 pacientes (ver tabla I).

\section{Colección de datos}

Durante el periodo de estudio (abril-junio 1999), además de determinar los niveles de glucosa en la sangre sin ayunar, se registraron otras variables relevantes para el estudio como la edad, el sexo, la altura, el peso (Se utilizó el Indice Quetelet: kg/m²) y el antipsicótico con el que era tratado el paciente en aquel momento.

\section{Análisis estadístico}

La prevalencia de hiperglicemia y DM, tipo 2 en la población se comparó con la prevalencia en la población general utilizando el test de Mantel-Haenzsel.

Se utilizó el test chi-cuadrado para evaluar los aspectos relativos a la prevalencia de la hiperglicemia en el grupo con sobrepeso comparado con lo que sucede en los de peso normal, así como para valorar la hiperglicemia y la DM tipo 2, en el caso de estar siendo tratados con antipsicóticos tradicio-

Tabla II

Non-fasting glucosa (capillary/venous)

Normoglicerina

Hyperglicerina

Diabetes Mellitus, tipo 2
Glucosa $<7,8 \mathrm{mmol} / 1$

$>$ 7,8 Glucosa $<11,0 \mathrm{mmol} / 1$

Glucosa > 11,0 mmol/1

Fuente: NHG Standard 1999. 
Tabla III

Valores estándar holandeses (*) en relación con la prevalencia de IGT y DM, tipo 2, en la población adulta:

\begin{tabular}{lccc} 
Edad (años) & \multicolumn{2}{c}{ Diabetes Mellitus tipo 2 } & IGT \\
& Estudio de Hoorn $(1995)^{39}$ & Nivel $(1999)^{40}$ & Estudio de Hoorn (1995) \\
\hline $20-39$ & $?(<2,6 \%)$ & $0,054 \%$ & $?(<5,7 \%)$ \\
$40-49$ & $?(<2,6 \%)$ & $0,736 \%$ & $?(<5,7 \%)$ \\
$50-54$ & $2,6 \%$ & $2,6 \%$ & $5,7 \%$ \\
$55-59$ & $6,1 \%$ & $2,6 \%$ & $7,0 \%$ \\
$60-64$ & $9,2 \%$ & $6,4 \%$ & $10,9 \%$ \\
$65-69$ & $11,2 \%$ & $6,4 \%$ & $13,1 \%$ \\
$70-74$ & $15,9 \%$ & $8,9 \%$ & $17,3 \%$ \\
\hline
\end{tabular}

nales, así como en relación con cada uno de tres antipsicóticos atípicos.

Los análisis estadísticos se valoraron con un nivel de significación del 5\% (bilateral)

El estudio se preparó sobre la base de los valores estándar NHG de 1999 para niveles de glucosa sin ayunar (ver tabla II).

Los datos resultantes del estudio, se compararon con los valores estándar procedentes de la literatura holandesa (ver tabla III).

El sistema que se utilizó para comparar las cifras de prevalencia requiere de una mayor explicación. Los valores estándar para una población general, se determinaron sobre la base del nivel de esfuerzo regular mediante un test de tolerancia a la glucosa por vía oral (OGTT), resultando una cifra de prevalencia IGT. En este estudio, era común la "inserción de glucosa”, por ejemplo: glucosa en vena o en capilares sin haber ayunado. Aquí, el nivel actual de glucosa no se conoce. Sin embargo, como máximo sería igual a 75 gramos de glucosa de OGTT, y en la mayoría de los casos sería mucho menor que éste. Debido a que la hiperglicemia se estableció sobre la base de la glucosa plasmática en sujetos no ayunadores, siendo ello específico no es lo bastante sensible como la IGT, por lo que la medida de la prevalencia para esta forma de hiperglicemia podría ser mas baja que la de la IGT. La distorsión resultante, probablemente le llevaría a una infraestimación del predominio de la hiperglicemia.

\section{Resultados}

\section{Hiperglicemia}

La hiperglicemia se descubrió en algunos de los 93 enfermos estudiados ( 7 hombres y 6 mujeres). En el 15,2\%, la prevalencia de hyperglicemia en pacientes con edades de entre 20 y 49 años fue significativo (OR = 2,959, CI = 1,230-7,119, $\mathrm{p}<0,05$ ) mientras que el porcentaje de IGT fue de un máximo del 5,7\% en el mismo grupo de edades de la población general (ver tabla III en el Estudio de Hoorn IGT). La cifra del 13,0\% de hiperglicemia de edades de entre 50 y 59 años, no fue significativamente mas alta que la de la prevalencia de la hiperglicemia en la población general (ver tabla III, Estudio de Hoorn, 50-54 años y 55-59 años) de un 6,3\% (OR = $2,220, \mathrm{CI}=, 0644-7,650, \mathrm{p}=, 206$ )

Para determinar la gravedad de la hiperglicemia, se intentó, un año después hacer el OGTT a esos 11 pacientes. Sin embargo, no se pudo realizar con 6 de ellos, ya que dos murieron por entonces, otros dos habían 
sido trasladados a otra Institución y otros dos rechazaron que se les tomara una muestra de sangre. Los resultados de la OGTT en los 5 restantes fueron un enfermo con normoglicemia, otro con hiperglicemia, y los tres restantes con Diabetes Mellitus, tipo 2.

\section{Diabetes Mellitus, tipo 2}

La prevalencia de DM, tipo 2, en esta población, se estableció en un $7,5 \%$, cifra que resultaba significativamente mas alta $(\mathrm{OR}=$ 4,288, CI = 1,979-9,289, $\mathrm{p}<0,001$ ) que la prevalencia de solo un $1,9 \%$ para la DM tipo 2 en la población general (Nivel 1999, ver tabla III). Para el tratamiento de la DM, tipo 2, en los pacientes esquizofrénicos, se utilizó la insulina en 5 de cada 7 pacientes $(70 \%)$ versus en el $10 \%$ de la población general.

\section{Factores de Riesgo}

\section{Edad}

No se encontraron diferencias significativas en cuanto a la edad entre los pacientes con normoglicemia, hiperglicemia o DM, tipo $2(\mathrm{~F}=, 368, \mathrm{df}=2,90, \mathrm{p}<, 693)$.

\section{Sexo}

No se hallaron diferencias significativas entre hombres y mujeres en relación con la prevalencia de la hiperglicemia (Chi-2 = ,967, $\mathrm{df}=1, \mathrm{p}=0,325)$ o DM, tipo 2 (Chi-2 $=0,12, \mathrm{df}=1, \mathrm{p}=, 911)$.

\section{Peso}

La prevalencia de obesos (QI>30) fue del $20,4 \%$, lo cual resultaba significativamente superior al $8 \%$ de prevalencia de la obesidad en la población general $(\mathrm{OR}=2,953, \mathrm{CI}=$ $1,698-5, \mathrm{p}<0,001)$. En enfermos que padecían de obesidad (QI>30), de hiperglicemia (Chi-2 = 3,203, df = 1, p < ,073) o DM, tipo $2($ Chi-2 $=2,342$, df $=1, \mathrm{p}=, 126)$ no se encontró que fuera ello claramente mas frecuente que otros enfermos.

\section{Utilización de los antipsicóticos: tradicionales y atípicos}

No vimos diferencias importantes entre los pacientes que recibían una medicación tradicional y los tratados con medicamentos atípicos, en relación con la presencia de hiperglicemia (Chi-2 $=1,241$, df $=1, \mathrm{p}=$ ,265) o DM tipo $2($ Chi-2 $=, 632, \mathrm{df}=1, \mathrm{p}=$ ,427). Tampoco existía ninguna relación establecida entre la utilización de los antipsicóticos tradicionales y el empleo de clozapina, risperidona o olanzapina de forma separada en relación con la prevalencia de hiperglicemia $($ Chi-2 $=1,728, \mathrm{df}=3, \mathrm{p}=, 631)$ o de DM, tipo $2($ Chi-2 $=1,154, \mathrm{df}=3, \mathrm{p}=, 766)$.

\section{Discusión}

En este estudio se ha mostrado como la prevalencia de hiperglicemia y DM, tipo 2 en una población que padece de esquizofrenia crónica, es significativamente mas alta que la que existe en la población general. Los factores de riesgo para contraer la hiperglicemia y la DM, tipo 2 son la edad, el sexo y el peso. El factor de riesgo diabetogénico en relación con otros medicamentos no se investigó debido al reducido número de pacientes involucrados, ya que únicamente dos enfermos utilizaban anticonceptivos por vía oral. A pesar de la alta prevalencia de obesidad, algo común en este grupo de 
pacientes, esta condición no fue un factor que explicara el incremento de la DM, tipo 2 o de la hiperglicemia. El que la influencia de una historia familiar de Diabetes Mellitus tipo 2 sea un factor de riesgo, parece no tener suficiente peso en estos casos debido a una serie de razones tales como la escasez de información existente en los archivos, la falta de contacto con las familias y la falta de cooperación por parte del paciente. En cuanto a los 7 enfermos que padecían de DM tipo 2 , esta información si que se encontraba disponible, pero en estos casos no existía DM tipo 2 en los parientes de primer grado.

Un aspecto importante de este estudio es el fuerte incremento de la prevalencia de hiperglicemia entre los esquizofrénicos crónicos menores de 50 años. En este estudio, 10 de cada 11 casos no habían sido diagnosticados previamente. A pesar del limitado tamaño de la muestra, el desarrollo de la hiperglicemia en el curso de un año, proporcionó una clara indicación acerca de la gravedad de la hiperglicemia en 3 de los 5 pacientes que desarrollaron DM, tipo 2. En un estudio reciente prospectivo en gran escala $(\mathrm{N}=25.364)$ de carácter longitudinal (7,3 años), la IGT mostró la tendencia a un mayor riesgo de muerte prematura de 1,51 $(1,32-1,72)$ en hombres y de 1,60 (1,222,10) en mujeres (DECODE 1999). Los enfermos que padecían de IGT, constituían un grupo de alto riesgo en relación con la muerte prematura, como sucede con los pacientes recientemente diagnosticados de DM: 1,81 (1,49-2,20) en hombres y 1,79 $(1,18-2,69)$ en mujeres.

Los resultados parecen confirmar los hallazgos de otros estudios epidemiológicopsiquiátricos, incluyendo los trabajos de Mukherjee y Hägg. En el estudio de Hägg, de acuerdo con nuestro trabajo, no se encontraron relaciones significativas entre la presencia de IGT o DM tipo 2 y el tipo de antipsicóticos empleados. Sin embargo, los hallazgos de Hägg se encontraban sujetos a una serie de limitaciones. Para empezar, se empleó el OGGT con un "drop-out" del $23 \%$ (en nuestro estudio fue del 6\%). En segundo lugar, la muestra de la población era, desde el punto de vista diagnóstico, bastante heterogénea y así junto a pacientes con esquizofrenia y trastornos psicoafectivos, se incluyeron otros cuadros psiquiátricos y entre ellos trastornos bipolares y trastornos de personalidad. En nuestro estudio la población estaba formada estrictamente por enfermos con esquizofrenia y trastornos esquizoafectivos. En tercer lugar, el número de pacientes que recibían antipsicóticos, tanto por vía oral como en depot, suponían mas del $50 \%$ en el estudio de Hägg (en nuestro estudio solo un 1\%). Además todos los pacientes que tomaban medicación depot (por ejemplo: fenotiazinas, butirofenonas y tioxantenos) se contaron como un grupo específico, sugiriendose que los efectos (cualitativos y cuantitativos) de los variados grupos químicos sobre el metabolismo de la glucosa era el mismo. Los resultados de los tests sobre animales han mostrado la gran variedad existente en cuanto a la influencia de los antipsicóticos sobre el metabolismo de la glucosa, incluso los pertenecientes al mismo grupo químico, por lo que no se justifica una clasificación tan amplia. Los mas recientes informes centrados en la clozapina (Kamran 1994, Ai 1997, Brugman 2000) o en la olanzapina (Goldstein 1999, Fertig 1998, Lindenmayer 1999) así como otros estudios a cerca de los antipsicóticos atípicos, en cuanto a la etiología de la Diabetes Mellitus (Hägg 1999, Melkersson 1999, Henderson 2000, Melkersson 2000) no pudieron confirmarse en nuestro estudio. Aunque sea consecuencia de diferencias en la población de estudio o se deba a otras diferencias metodológicas, creemos 
que todo ello debería ser objeto de una investigación mas profunda.

Este estudio cuenta con tres grandes limitaciones, una es el impacto que supone la inactividad de estos pacientes, factor de riesgo éste bien conocido en el caso de la Diabetes Mellitus tipo 2 y que no se valoró. La segunda limitación tiene que ver con la selección de la población de estudio, ya que únicamente se incluyeron enfermos esquizofrénicos crónicos e internados. La tercera limitación fue la dificultad para poder valorar el efecto de la cronicidad, con medicaciones antipsicóticas administradas a dosis muy variadas. Se necesitan por ello mas investigaciones para esclarecer estos aspectos.

\section{Conclusión}

En un estudio exploratorio sobre 93 esquizofrénicos crónicos, las prevalencias de la DM, tipo 2 y de la hiperglicemia en pacientes menores de 50 años parecía ser significativamente mas alta que la de la población general. No se halló conexión alguna entre factores de riesgo tales como el sexo, la edad, la DM tipo 2 o la hiperglicemia. Aunque habían muchos obesos en la población estudiada, no se encontró conexión alguna entre la presencia de DM tipo 2 o hiperglicemia y la obesidad. Igualmente, no se encontró conexión entre la prevalencia de DM o hiperglicemia y los antipsicóticos prescritos, como tampoco al tener en cuenta a los dos grupos de antipsicóticos (el tradicional y el atípico) o incluso al tener en cuenta cada uno de los medicamentos atípicos por separado.

Los resultados del estudio corroboraron algunos resultados de otros estudios, mostrando el incremento de las prevalencias de
DM tipo 2 y de hiperglicemia en los pacientes esquizofrénicos, pero no confirmando los recientes estudios sobre las acciones de los antipsicóticos atípicos y tradicionales, en relación con la Diabetes Mellitus tipo 2.

Es necesario un programa epidemiológico a gran escala (pacientes externos regulares, pacientes en cuidados intensivos en la comunidad, y pacientes internos de larga estancia) para responder a la cuestión de si la condición de padecer de esquizofrenia por sí misma constituye un factor de riesgo (Maudsley, 1899, Mukherjee 1989) o si ella solo contribuye de forma indirecta a través del desarrollo de algunas otras condiciones de riesgo como la obesidad, la inactividad, la ingestión de alimentos no saludables para la Diabetes Mellitus, o si es el efecto diabetogénico directo de los antipsicóticos el que realmente está en juego.

\section{Bibliografía}

AMDISEN, A. Diabetes mellitus as a side effect of treatment with tricyclic neuroleptics. Acta Psychiatrica Scandinavica, 180, 411-414, 1964.

AMMON, H.P.T., ORCI, L., STEINKE, J. Effect of chlorpromazine (CPZ) on insulin release in vivo and in vitro in the rat. Journal of Pharmacology and Experimental Therapeutics, 187, 423-429, 1973.

BERGHOUT, L., GORTER, K., RUTTEN, G. DIATWINSonderzoek Apeldoorn 1999 (in preparation). Quoted in text as NIVEL 1999.

BHIDE, M.B., TIWARI, N.M., BALWANI, J.H. Effect of chlorpromazine on peripheral utilisation of glucose. Archives of International Pharmacodynamics, 156, 166-171, 1965.

BONACCORSI, A., GARATTINI, S., JORI, A. Studies on the hyperglycaemia induced by chlorpromazine in rats. British Journal of Pharmacology, 23, 93-100, 1964.

BRUGMAN, N.J., COHEN, D., DE VRIES, R.H. Diabetes ontstaan na behandeling met clozapine. Nederlands Tijdschrift voor Geneeskunde, 144, 437-439, 2000. 
CHARATAN, F.B.E., BARTLETT, N.G. The effect of chlorpromazine ("Largactil") on glucose tolerance. Journal of Mental Science, 101, 351-353, 1955.

CLAYER, J.R., DUMBRILL, M.N. Diabetes mellitus and mental illness. Med. J. Austr. I: 901-904, 1967.

COLLI, A., COCCIOLO, M., FRANCOBANDIERA, G., ROGANTIN, F., CATTALINI, N. Diabetic ketoacidosis associated with clozapine treatment. Diabetes Care, 22, 176-177, 1999.

DAWN, A.T., ROPER, T.A., RILEY, J.A. Diabetic ketoacidosis and clozapine. Adverse Drug Reactions, 493-494, 1997.

DECODE study group on behalf of the European Diabetes Epidemiology Group. Glucose tolerance and mortality: comparison of WHO and American Diabetes Association diagnostic criteria Lancet 354: 617-621, 1999.

DELAY, J., DENIKER, P., HARL, J.M. et al. Traitements d'états confussionels par le chlorhydrate de diéthylaminopropyl-N-chlorophénothiazine (4560 RP). Annales Medico-Psychologiques, 110, 398-403, 1952.

FERTIG, M.K., BROOKS, V.G., SHELTON, P.S. Hyperglycaemia associated with olanzapine. Journal of Clinical Psychiatry, 59, 687-689, 1998.

GHAFGHAZI, T., MIYA, T.S., MENNEAR, J.H., CHALMERS, R.K. Chlorpromazine and epinephrine hyperglycemic mechanisms. Journal of Pharmaceutical Sciences, 57, 1690-1693, 1968.

GOLDSTEIN, L.E., SPORN, J., BROWN, S., KIM, H., FINKELSTEIN, J., GAFFEY, G.K., SACHS, G., STERN, T.A. New-onset diabetes mellitus and diabetic ketoacidosis associated with olanzapine treatment. Psychosomatics, 40, 438-443, 1999.

HÄGG, S., JOELSSON, L., MJORNDAL, T., SPIGSET, O., OJA, G., DAHLQVIST, R. Prevalence of diabetes and impaired glucose tolerance in patients with clozapine compared with patients treated with conventional depot neuroleptic medications. Journal of Clinical Psychiatry, 294299, 1998.

HEIDEMA, S.T. Bloedsuikerbepalingen bij psychiatrische en neurologische patiënten. Dissertatie, Utrecht, 1918.

HILES, BW. Hyperglycaemia and glycosuria following chlorpromazine therapy. Journal of American Medical Association, 162, 1651, 1956.

Inspectie voor de Gezondheidszorg. Somatische zorg in APZ'en. Den Haag: Inspectie, 1999.

JORI, A., CARRARA, M.C. On the mechanism of the hyperglycaemic effect of chlorpromazine. Journal of Pharmacy and Pharmacology, 18, 623-624, 1966.
KAMRAN, A., DORAISWAMY, P.M., JANE, J.L., HAMMETT, E.B., DUNN, L. Severe hyperglycaemia associated with high doses of clozapine. American Journal of Psychiatry, 151, 1395, 1994.

KESKINER, A., EL TOUMI, A., BOUSQUET, T. Psychotropic drugs, diabetes and chronic mental illness. Psychosomatics, 14: 176-181, 1973.

KOOY, F.H. Hyperglycaemia in mental disorders. Brain, 42, 213-289, 1919.

LINDENMAYER, J.-P., PATEL, R. Olanzapine-induced ketoacidosis with diabetes mellitus. American Journal of Psychiatry, 156: 1471, 1999 .

McFARLAND, R.A., GOLDSTEIN, H. The biochemistry of dementia praecox. American Journal of Psychiatry, 95, 509-552, 1938.

MELKERSSON, K.I., HULTING, A.-L., BRISMAR, K.E. Different influences of classical antipsychotics and clozapine on glucose-insulin homeostasis in patients with schizophrenia or related psychosis. Journal of Clinical Psychiatry, 60, 783-791, 1999.

MELKERSSON, K.I., HULTING, A.-L., BRISMAR, K.E. Elevated levels of insulin, leptin, and blood lipids in olanzapine-treated patients with schizophrenia or related psychosis. Journal of Clinical Psychiatry, 61, 742-749, 2000.

MELTZER, H.Y. The concept of an atypical anti-psychotic drug. In: Boer, J.A. den, Westenberg, H.G.M., Praag, H.M. van, (Eds.) Advances in the neurobiology of schizophrenia. Chicester. Wiley \& Sons, 1995.

MOOY, J.M. Non-Insulin-Dependent Diabetes Mellitus in an General Caucasian Population The Hoorn Study. Proefschrift, Amsterdam. Quoted in text as Hoorn Study, 1995.

MUKHERJEE, S., SCHNUR, D.B., REDDY, R. Family history of type 2 diabetes in schizophrenic patients [letter]. Lancet, 495, 1989.

MUKHERJEE, S. High prevalence of type II diabetes mellitus in schizophrenic patients. Schizophrenia Research, 15, 195, 1995.

MUKHERJEE, S., DECINA, P., BOCOLA, V., SARACENI, F., SCAPICCHIO, P.L. Diabetes mellitus in schizophrenic patients. Comprehensive Psychiatry, 37, 68-73, 1996.

Nederlands Huisartsen Genootschap-standaard Diabetes Mellitus type 2. Huisarts en Wetenschap 1999; 42: $67-$ 74. Quoted in text as NHG-standard.

PROAKIS, A.G., MENNEAR, J.H., MIYA, T.S., BOROWITZ, J.L. Phenothiazine-induced hyperglycaemia: relation to CNS and adrenal effects. Proceedings Society Experimental Biology and Medicine, 137, 1385-1388, 1971. 
SCHWARZ, L., MUNOZ, R. Blood sugar levels in patients treated with chlorpromazine. American Journal of Psychiatry, 125, 253-255, 1968.

THONNARD-NEUMANN, E. Phenothiazines and diabetes in hospitalized women. American Journal of Psychiatry, 124, 138-142, 1968.

WAITZKIN, L. A survey for unknown diabetics in a mental hospital. 1. Men under age fifty. Diabetes, 6, 97-104, 1966.

WAITZKIN, L. A survey for unknown diabetics in a mental hospital. 2. Men from age fifty. Diabetes, 15, 164$172,1966$.

WANNARKA, G.I., FLETCHER, H.P., MAICKEL, R.P. Centrally mediated drug-induced hyperglycaemia in mice. Neuropharmacology, 22, 341-346, 1983.
WIRSHING, D.A., SPELLBERG, B.J., ERHART, S.M., MARDER, S.R., WIRSHING, W.C. Novel anti-psychotics and new onset diabetes. Biological Psychiatry, 44, 778-783, 1998.

Dirección para correspondencia: Dan Cohen Director del Departamento de Largas Estancias (Rijngeestgroep)

Langevelderweg 27

2211 AB Noordwijkerhout HOLANDA 